\title{
In vitro regeneration of Drosera burmannii Vahl.: a carnivorous plant of north-east India
}

\author{
J. Sureni Yanthan ${ }^{1} \cdot$ Mechuselie Kehie $^{2,3} \cdot$ Suman Kumaria $^{1} \cdot$ Pramod Tandon $^{1}$
}

Received: 1 September 2016/Accepted: 14 February 2017/Published online: 31 May 2017

(C) Springer-Verlag Berlin Heidelberg 2017

\begin{abstract}
An efficient in vitro regeneration protocol has been developed from shoot tips of Drosera burmannii Vahl., a carnivorous plant of north-east India. Various plant growth regulators were used to study their efficacy in the induction of multiple shoots and roots. Of the various treatments, the maximum number of shoots $(28.8 \pm 1.5)$ and roots $(9.7 \pm 0.6)$ was observed in one-fourth strength standard medium (MS with $50 \mathrm{mg} / \mathrm{l}$ citric acid and $10 \mathrm{mg} / \mathrm{l}$ ascorbic acid) supplemented with $4 \mathrm{mg} / \mathrm{l}$ 6-benzylaminopurine (BAP) and $4 \mathrm{mg} / \mathrm{l} \alpha$-naphthalene acetic acid (NAA) followed by $26.8 \pm 1.4$ shoots in one-fourth strength SM fortified with $4 \mathrm{mg} / \mathrm{l}$ kinetin $(\mathrm{KN})$ and $4 \mathrm{mg} / \mathrm{l}$ NAA. The well-developed plantlets with shoots and roots were potted in small plastic glasses filled with a mixture of sand and farmyard manure (3:1); these plantlets when transferred to a glasshouse for hardening and acclimatization showed $90 \%$ survival.
\end{abstract}

Keywords Carnivorous plant · Drosera burmannii · In vitro regeneration

Mechuselie Kehie

kehie10@gmail.com

1 Plant Biotechnology Laboratory, Centre for Advanced Studies in Botany, North-Eastern Hill University, Shillong 793022, India

2 Post Graduate Department of Environmental Science, Patkai Christian College (Autonomous), Chumukedima, Seithekema, Dimapur 797103, India

3 Sustainable Development Research Centre, Kohima 797001, Nagaland, India

\section{Introduction}

Charles Darwin described carnivorous plants as 'the most wonderful plants in the world'. Carnivorous plants show a novel way of adaptation to survive in nutrientdeficient environments. Although they follow different ways of adaptations with varying complexities, the carnivorous plants serve the same purpose. The genus Drosera belonging to the family Droseraceae is commonly known as sundew. The sundew is considered as the master of sticky flypaper due to the presence of long tentacles on their leaves which act as a carnivory to catch prey. The word Drosera is derived from the Greek word Drosero which means 'glistening in the sun or dewy'. There are more than 200 known species which fall under this genus (McPherson 2010).

Drosera burmannii Vahl., usually found in acidic and sandy soils, is a tropical sundew or Burmese sundew annual carnivorous plant. It is an erect herb with short stem and rosette leaves. Each leaf is $8-10 \mathrm{~mm}$ long and 5-6 mm wide. The flowers are white in color, arising on a leafless scape measuring about $5-20 \mathrm{~cm}$ long. The plant grows to a height of about $5 \mathrm{~cm}$. Because of their carnivorous nature and the beauty of their glistening traps, sundews have become favorite ornamental plants. D. burmannii is geographically distributed in Southeast Asia, China, India, Japan, West Africa and Australia (Nordbakken et al. 2004). The major compounds found in D. burmannii are naphthoquinones, plumbagin, ramanteeon, glucoside rossoliside, flavonoids like quercetin and hyperoside (Wagner et al. 1984; Wang et al. 1998). The plant also exhibits anticonvulsant activity (Hema et al. 2009) and rubefacient property. The locals of Khasi and Jaintia Hills of Meghalaya, India, have reported the usage of these leaves mixed with salt to remove or heal blisters (Joseph and Joseph 
1986). This plant also forms a vital component in an Ayurvedic preparation called 'Swarnabhasma' (Golden ash) (Ravikumar et al. 2000). In many European countries and China, D. burmannii are imported to pharmaceutical industries, where the dried plants are marketed as 'Herba Droserae' (Didry et al. 1998). Apart from obtaining nutrients from animals, carnivorous plants sometimes show a complex interaction with them (Jennings et al. 2010; Moon et al. 2010). Carnivorous plants consume pests such as mosquitoes, midges, deer flies, horseflies and dipterans which transmit human diseases (Ellison and Gotelli 2009). Consequently, the conservation of carnivorous plants could benefit many other taxa including humans. It also helps in preventing secondary extinction of other specialist species which rely on them. As the collection of the plants increases, it ultimately leads to decline in wild populations. D. burmannii has been tagged as vulnerable in India (Reddy et al. 2001), and in the state of Andhra Pradesh the plant is one of the Red Listed medicinal insectivorous plants (Jayaram and Prasad 2006). Multiplication and conservation through tissue culture technique forms one of the major methods of protecting the species. Such conservation efforts would ensure continuous and ample supply of this valuable material which is in great demand by the pharmaceutical industries. In the present study, we aim to establish an efficient micropropagation protocol for D. burmannii, using different cultural strategies so as to help in the conservation and propagation of this plant species.

\section{Materials and methods}

Drosera burmannii Vahl. were collected from Jarain, Meghalaya, and maintained in a glasshouse at the Plant Biotechnology Laboratory, Department of Botany, North Eastern Hill University, Shillong. Shoot tips were harvested from the plants growing in the glasshouse and used as explants. Explants were washed thoroughly in running tap water and then treated with $10 \%$ sodium hypochlorite (4\% active chlorine content) for $10 \mathrm{~min}$ and finally rinsed six times with distilled water. The shoot tips measuring about $1 \mathrm{~cm}^{2}$ were inoculated on one-fourth strength standard medium (MS with $50 \mathrm{mg} / \mathrm{l}$ citric acid and $10 \mathrm{mg} / \mathrm{l}$ ascorbic acid), containing 3\% (w/v) sucrose and $0.8 \%(\mathrm{w} /$ v) agar (Himedia), and the $\mathrm{pH}$ was adjusted to 5.8. All media were autoclaved at $121{ }^{\circ} \mathrm{C}$ and $1.05 \mathrm{~kg} \mathrm{~cm}^{-2}$ pressure for $15 \mathrm{~min}$. Cultures were incubated in the growth room at a temperature of $25 \pm 2{ }^{\circ} \mathrm{C}, 14 / 10$-h photoperiod with an irradiance of $60 \mu \mathrm{mol} \mathrm{m} \mathrm{m}^{-2} \mathrm{~s}^{-1}$ provided by cool white fluorescent tubes.

\section{Induction of shoots and roots}

For induction of shoots and roots, shoot tip explants were excised from the in vitro raised microshoots and subcultured in different strengths of SM supplemented with varying concentrations (1-8 mg/l) of 6-benzylaminopurine (BAP), (Z) and kinetin (KN) in combination with $\alpha$ naphthalene acetic acid (NAA) (Fig. 1; Tables 1, 2, 3). Data for organogenetic response and shoot multiplication were recorded after 30 days of culture inoculation. The number of roots formed was recorded after 30 days of microshoot transfer. All experiments were repeated thrice with six replicates each and data were analyzed using oneway analysis of variance (ANOVA) in $\mathrm{JMP}^{\circledR}$ version 7.0.1 (SAS Institute, Cary, NC). The significant differences among the means were assessed by Tukey's test at $5 \%$ probability level.

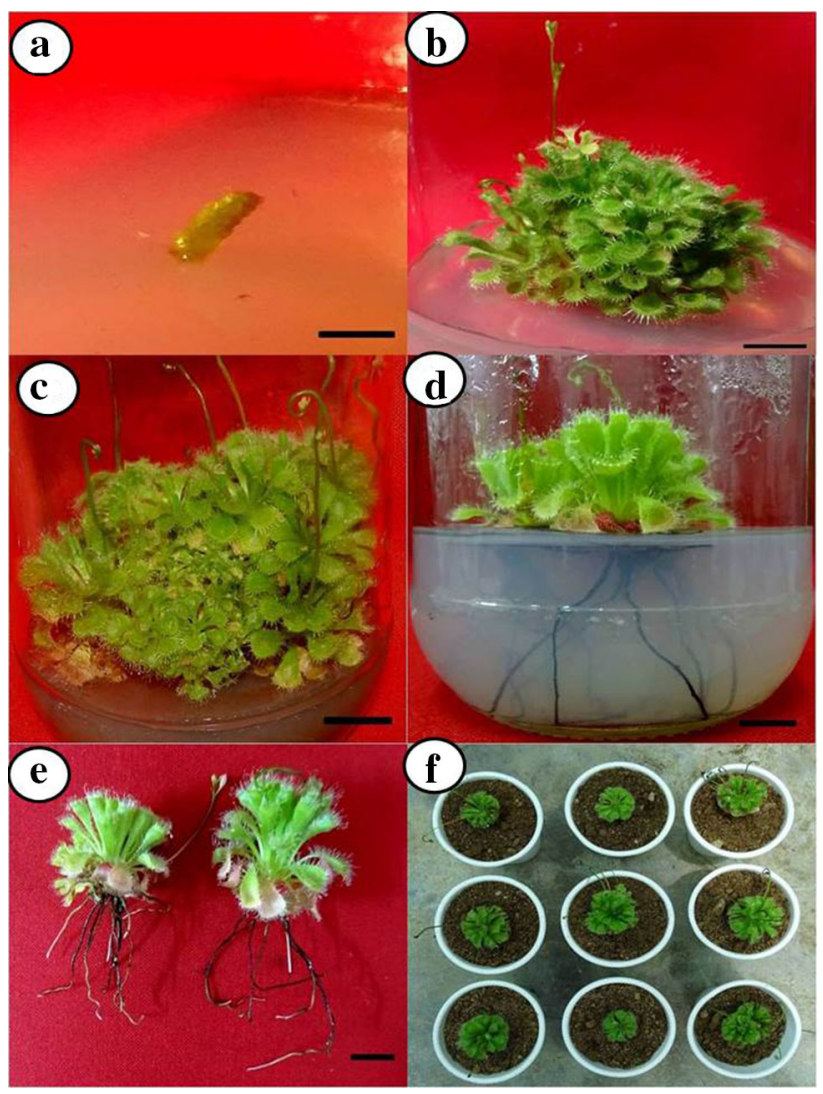

Fig. 1 Multiple shoot formation and root induction from shoot tip explants of D. burmannii. a Initial stage of inoculation of shoot tip explants $($ Bar $1 \mathrm{~cm})$. b Multiple shoot induction in one-fourth strength $\mathrm{SM}+\mathrm{BAP}+\mathrm{NAA}(4 \mathrm{mg} / \mathrm{l})$ after 30 days $($ Bar $1 \mathrm{~cm})$. c Multiple shoot induction in one-fourth strength $\mathrm{SM}+\mathrm{BAP}+$ NAA $(4 \mathrm{mg} / \mathrm{l})($ Bar $1 \mathrm{~cm})$. d Root induction in one-fourth strength $\mathrm{MS}+\mathrm{BAP}+\mathrm{NAA}(4 \mathrm{mg} / \mathrm{l})$ after 30 days $($ Bar $1 \mathrm{~cm})$. e Welldeveloped roots $($ Bar $1 \mathrm{~cm})$. f Regenerated hardened plantlets 
Table 1 Adventitious responses of Drosera burmannii Vahl. from shoot tip explants cultured in various strengths of SM supplemented with different concentrations of BAP and NAA

\begin{tabular}{|c|c|c|c|c|c|c|c|}
\hline \multirow{2}{*}{\multicolumn{2}{|c|}{ PGR (mg/l) }} & \multicolumn{6}{|c|}{ After 30 days of culture } \\
\hline & & \multicolumn{2}{|c|}{ Full-strength SM } & \multicolumn{2}{|l|}{$1 / 2$ Strength SM } & \multicolumn{2}{|l|}{ 1/4 Strength SM } \\
\hline BAP & NAA & No. of shoots & No. of roots & No. of shoots & No. of roots & No. of shoots & No. of roots \\
\hline \multicolumn{2}{|l|}{ Control } & $15.3 \pm 1.1$ & $1.5 \pm 0.3$ & $15.5 \pm 1.2$ & $2.1 \pm 0.2$ & $17.8 \pm 1.3$ & $5.3 \pm 0.3$ \\
\hline 1 & 1 & $16.1 \pm 1.2$ & $2.1 \pm 0.2$ & $15.6 \pm 0.8$ & $3.5 \pm 0.1$ & $20.9 \pm 1.2^{*}$ & $5.2 \pm 0.4$ \\
\hline 1 & 2 & $16.3 \pm 0.4$ & $2.3 \pm 0.4$ & $17.2 \pm 1.1 *$ & $6.3 \pm 0.4^{*}$ & $21.5 \pm 1.6^{*}$ & $5.5 \pm 0.2$ \\
\hline 1 & 4 & $18.6 \pm 0.8^{*}$ & $3.1 \pm 0.5^{*}$ & $18.5 \pm 1.5^{*}$ & $4.5 \pm 0.3^{*}$ & $22.6 \pm 1.3^{*}$ & $5.4 \pm 0.5$ \\
\hline 1 & 8 & $16.3 \pm 0.9$ & $2.2 \pm 0.2$ & $14.7 \pm 1.2$ & $3.6 \pm 0.5^{*}$ & $23.3 \pm 1.6^{*}$ & $6.6 \pm 0.4$ \\
\hline 2 & 1 & $16.6 \pm 0.6$ & $1.8 \pm 0.7$ & $16.3 \pm 2.3$ & $2.1 \pm 0.7$ & $19.3 \pm 1.4$ & $6.7 \pm 0.4$ \\
\hline 2 & 2 & $15.6 \pm 0.7$ & $1.6 \pm 0.6$ & $17.6 \pm 2.4^{*}$ & $2.2 \pm .0 .5$ & $20.2 \pm 1.6^{*}$ & $6.4 \pm 0.5$ \\
\hline 2 & 4 & $15.3 \pm 0.3$ & $1.4 \pm 0.4$ & $16.3 \pm 1.1$ & $2.4 \pm 0.6$ & $22.6 \pm 1.2 *$ & $6.2 \pm 0.6$ \\
\hline 2 & 8 & $14.6 \pm 0.7$ & $1.2 \pm 0.8$ & $13.2 \pm 1.3$ & $2.5 \pm 0.2$ & $23.4 \pm 1.5^{*}$ & $7.1 \pm 0.6^{*}$ \\
\hline 4 & 1 & $16.1 \pm 0.2$ & $1.1 \pm 0.2$ & $22.4 \pm 1.4 *$ & $2.7 \pm 0.4$ & $25.7 \pm 1.6^{*}$ & $7.3 \pm 0.3^{*}$ \\
\hline 4 & 2 & $17.6 \pm 0.8^{*}$ & $1.1 \pm 0.3$ & $26.2 \pm 1.8^{*}$ & $1.5 \pm 0.7$ & $26.3 \pm 1.1 *$ & $8.2 \pm 0.5^{*}$ \\
\hline 4 & 4 & $16.3 \pm 0.7$ & $1 \pm 0.2$ & $25.2 \pm 1.4^{*}$ & $1.4 \pm 0.2$ & $28.8 \pm 1.5^{*}$ & $9.7 \pm 0.6^{*}$ \\
\hline 4 & 8 & $15.3 \pm 0.8$ & $1 \pm 0.2$ & $23.7 \pm 1.6^{*}$ & $1.2 \pm 0.3$ & $25.9 \pm 0.9 *$ & $7.5 \pm 0.2^{*}$ \\
\hline 8 & 1 & $16.3 \pm 0.9$ & - & $20.7 \pm 2.1^{*}$ & $1.3 \pm 0.4$ & $23.4 \pm 1.2 *$ & $5.3 \pm 0.5$ \\
\hline 8 & 2 & $15.4 \pm 0.5$ & - & $18.4 \pm 1.7^{*}$ & $1.5 \pm 0.2$ & $22.2 \pm 0.9 *$ & $4.2 \pm 0.6$ \\
\hline 8 & 4 & $14.5 \pm 0.7$ & - & $17.1 \pm 2.1 *$ & $1.3 \pm 0.4$ & $20.1 \pm 1.4^{*}$ & $4.1 \pm 0.2$ \\
\hline 8 & 8 & $14.1 \pm 0.8$ & - & $15.4 \pm 1.5$ & $1.2 \pm 0.5$ & $18.7 \pm 1.2$ & $4.2 \pm 0.1$ \\
\hline
\end{tabular}

Mean \pm SE

* Significant difference among treatments and control $(P<0.05)$

Table 2 Adventitious responses of Drosera burmannii Vahl. from shoot tip explants cultured in various strengths of SM supplemented with different concentrations of KN and NAA

\begin{tabular}{|c|c|c|c|c|c|c|c|}
\hline \multirow{2}{*}{\multicolumn{2}{|c|}{ PGR (mg/l) }} & \multicolumn{6}{|c|}{ After 30 days of culture } \\
\hline & & \multicolumn{2}{|c|}{ Full-strength SM } & \multicolumn{2}{|c|}{$1 / 2$ Strength SM } & \multicolumn{2}{|c|}{ 1/4 Strength SM } \\
\hline $\mathrm{KN}$ & NAA & No. of shoots & No. of roots & No. of shoots & No. of roots & No. of shoots & No. of roots \\
\hline Control & & $15.3 \pm 1.1$ & $1.5 \pm 0.3$ & $15.5 \pm 1.2$ & $2.1 \pm 0.2$ & $17.8 \pm 1.3$ & $5.3 \pm 0.3$ \\
\hline 1 & 1 & $15.1 \pm 1.9$ & - & $13.6 \pm 0.9$ & $2.3 \pm 0.5$ & $12.6 \pm 1.8$ & $5.1 \pm 0.9$ \\
\hline 1 & 2 & $16.9 \pm 0.7$ & - & $17.9 \pm 1.3^{*}$ & $2.5 \pm 0.6$ & $18.3 \pm 1.8$ & $6.3 \pm 0.6$ \\
\hline 1 & 4 & $17.6 \pm 0.7^{*}$ & - & $16.4 \pm 1.9$ & $2.6 \pm 0.4$ & $19.3 \pm 1.9^{*}$ & $7.2 \pm 0.2^{*}$ \\
\hline 1 & 8 & $16.7 \pm 0.5$ & - & $13.3 \pm 1.8^{*}$ & $2.8 \pm 0.7$ & $17.3 \pm 1.7$ & $5.8 \pm 0.4$ \\
\hline 2 & 1 & $18.6 \pm 0.4^{*}$ & $1.4 \pm 0.3$ & $14.8 \pm 1.2$ & $3.2 \pm 0.9$ & $16.4 \pm 1.8$ & $5.6 \pm 0.6$ \\
\hline 2 & 2 & $20.4 \pm 0.9^{*}$ & $2.2 \pm 0.5$ & $16.3 \pm 1.4$ & $3.3 \pm 0.4$ & $17.3 \pm 1.8$ & $5.4 \pm 0.4$ \\
\hline 2 & 4 & $18.6 \pm 0.8^{*}$ & $1.4 \pm 0.5$ & $14.8 \pm 1.3$ & $3.6 \pm 0.2 *$ & $19.3 \pm 1.2^{*}$ & $5.3 \pm 0.9$ \\
\hline 2 & 8 & $19.4 \pm 0.6^{*}$ & $1.3 \pm 0.2$ & $12.9 \pm 1.1^{*}$ & $3.4 \pm 0.4$ & $21.6 \pm 1.8^{*}$ & $5.1 \pm 0.5$ \\
\hline 4 & 1 & $17.1 \pm 0.7$ & $3.2 \pm 0.6^{*}$ & $20.3 \pm 1.3^{*}$ & $3.7 \pm 0.6^{*}$ & $22.1 \pm 1.8^{*}$ & $4.8 \pm 0.8$ \\
\hline 4 & 2 & $14.7 \pm 0.5$ & $1.5 \pm 0.3$ & $24.7 \pm 1.7^{*}$ & $3.9 \pm 0.3^{*}$ & $24.4 \pm 1.1^{*}$ & $4.5 \pm 0.5$ \\
\hline 4 & 4 & $13.5 \pm 0.8$ & $1.3 \pm 0.2$ & $23.3 \pm 1.8^{*}$ & $3.6 \pm 0.8^{*}$ & $26.3 \pm 1.4^{*}$ & $4.3 \pm 0.3$ \\
\hline 4 & 8 & $12.2 \pm 0.7^{*}$ & $1.3 \pm 0.1$ & $22.5 \pm 1.7^{*}$ & $3.4 \pm 0.6$ & $24.2 \pm 1.7^{*}$ & $4.1 \pm 0.4$ \\
\hline 8 & 1 & $14.7 \pm 0.8$ & - & $20.7 \pm 1.6^{*}$ & $3.3 \pm 0.4$ & $23.6 \pm 1.8^{*}$ & $3.6 \pm 0.9^{*}$ \\
\hline 8 & 2 & $16.4 \pm 0.4$ & - & $17.2 \pm 1.8$ & $2.6 \pm 0.3$ & $22.3 \pm 1.9^{*}$ & $3.4 \pm 0.5^{*}$ \\
\hline 8 & 4 & $15.7 \pm 0.6$ & - & $14.8 \pm 1.7$ & $2.4 \pm 0.2$ & $20.1 \pm 1.6^{*}$ & $3.2 \pm 0.3^{*}$ \\
\hline 8 & 8 & $13.1 \pm 0.6^{*}$ & - & $12.4 \pm 1.7^{*}$ & $2.2 \pm 0.1$ & $18.7 \pm 1.9$ & $2.3 \pm 0.2^{*}$ \\
\hline
\end{tabular}

Mean \pm SE

* Significant difference among treatments and control $(P<0.05)$ 
Table 3 Adventitious responses of Drosera burmannii Vahl. from shoot tip explants cultured in various strengths of SM supplemented with different concentrations of $\mathrm{Z}$ and NAA

\begin{tabular}{|c|c|c|c|c|c|c|c|}
\hline \multirow{2}{*}{\multicolumn{2}{|c|}{ PGR (mg/l) }} & \multicolumn{6}{|c|}{ No. of shoots after 30 days } \\
\hline & & \multicolumn{2}{|c|}{ Full-strength SM } & \multicolumn{2}{|c|}{$1 / 2$ Strength SM } & \multicolumn{2}{|c|}{ 1/4 Strength SM } \\
\hline $\mathrm{Z}$ & NAA & No. of shoots & No. of roots & No. of shoots & No. of roots & No. of shoots & No. of roots \\
\hline \multicolumn{2}{|c|}{ Control } & $15.3 \pm 1.1$ & $1.5 \pm 0.3$ & $15.5 \pm 1.2$ & $2.1 \pm 0.2$ & $17.8 \pm 1.3$ & $5.3 \pm 0.3$ \\
\hline 1 & 1 & $13.1 \pm 1.2^{*}$ & - & $10.6 \pm 1.5 *$ & $1.6 \pm 0.6$ & $18.6 \pm 1.3$ & $3.2 \pm 0.6^{*}$ \\
\hline 1 & 2 & $14.9 \pm 1.7$ & - & $11.3 \pm 1.4 *$ & $1.8 \pm 0.4$ & $20.3 \pm 1.3 *$ & $4.5 \pm 0.7$ \\
\hline 1 & 4 & $16.8 \pm 1.1$ & - & $13.2 \pm 1.1 *$ & $2.1 \pm 0.5$ & $23.5 \pm 1.7 *$ & $5.1 \pm 0.4$ \\
\hline 1 & 8 & $18.8 \pm 1.8^{*}$ & - & $11.4 \pm 1.3 *$ & $2.3 \pm 0.3$ & $25.3 \pm 1.2 *$ & $5.3 \pm 0.6$ \\
\hline 2 & 1 & $22.6 \pm 1.7^{*}$ & $2.7 \pm 0.2$ & $15.8 \pm 1.4$ & $2.6 \pm 0.8$ & $19.4 \pm 1.8$ & $6.4 \pm 0.3$ \\
\hline 2 & 2 & $24.5 \pm 1.5^{*}$ & $3.4 \pm 0.3^{*}$ & $17.3 \pm 1.3$ & $3.2 \pm 0.5$ & $22.3 \pm 1.3^{*}$ & $7.2 \pm 0.6^{*}$ \\
\hline 2 & 4 & $15.1 \pm 0.3$ & $1.6 \pm 0.4$ & $20.8 \pm 1.7 *$ & $4.1 \pm 0.6^{*}$ & $21.3 \pm 1.4 *$ & $6.3 \pm 0.2$ \\
\hline 2 & 8 & $13.6 \pm 1.5$ & $1.8 \pm 0.6$ & $18.9 \pm 1.8^{*}$ & $3.1 \pm 0.3$ & $22.6 \pm 1.7 *$ & $5.3 \pm 0.8$ \\
\hline 4 & 1 & $12.4 \pm 1.8^{*}$ & $1.5 \pm 0.8$ & $15.3 \pm 1.7$ & $2.7 \pm 0.2$ & $19.1 \pm 1.7$ & $4.2 \pm 0.7$ \\
\hline 4 & 2 & $11.8 \pm 1.6^{*}$ & $1.3 \pm 0.3$ & $18.9 \pm 1.8^{*}$ & $2.6 \pm 0.8$ & $22.4 \pm 1.8^{*}$ & $3.7 \pm 0.5^{*}$ \\
\hline 4 & 4 & $13.8 \pm 1.6$ & $1.2 \pm 0.3$ & $15.3 \pm 1.3$ & $2.5 \pm 0.9$ & $17.3 \pm 1.9$ & $3.7 \pm 0.4^{*}$ \\
\hline 4 & 8 & $11.8 \pm 1.8^{*}$ & $1.1 \pm 0.1$ & $15.5 \pm 1.8$ & $2.1 \pm 0.5$ & $15.9 \pm 1.2$ & $3.2 \pm 0.3^{*}$ \\
\hline 8 & 1 & $10.8 \pm 0.9^{*}$ & - & $13.8 \pm 1.3$ & $1.9 \pm 0.3$ & $18.6 \pm 1.5$ & $2.8 \pm 0.2^{*}$ \\
\hline 8 & 2 & $12.6 \pm 1.7^{*}$ & - & $16.2 \pm 1.1$ & $1.6 \pm 0.1$ & $22.9 \pm 1.3^{*}$ & $2.6 \pm 0.1^{*}$ \\
\hline 8 & 4 & $11.8 \pm 1.6^{*}$ & - & $13.8 \pm 1.6$ & $1.3 \pm 0.3$ & $21.1 \pm 1.8^{*}$ & $2.5 \pm 0.8^{*}$ \\
\hline 8 & 8 & $10.1 \pm 1.9^{*}$ & - & $11.4 \pm 1.8^{*}$ & $1.1 \pm 0.4$ & $18.9 \pm 1.4$ & $2.2 \pm 0.5^{*}$ \\
\hline
\end{tabular}

Mean \pm SE

* Significant difference among treatments and control $(P<0.05)$

\section{Acclimatization and transplantation}

The in vitro rooted plantlets were washed thoroughly and potted in small plastic glasses filled with a mixture of sand and farmyard manure (3:1) and irrigated with water daily in a glasshouse. The plantlets were maintained under glasshouse condition at a temperature of $25 \pm 2{ }^{\circ} \mathrm{C}, 80 \%$ relative humidity, photon flux of $100 \mathrm{~mol} \mathrm{~m}{ }^{-2} \mathrm{~s}^{-1}$ and a day length of $12 \mathrm{~h}$. The average survival percentage was calculated after 4 weeks of acclimatization.

\section{Results and discussions}

Plant growth regulators (PGR) have intense effect on seedling growth of many plants (Su et al. 2011). PGR cytokinins enhance shoot regeneration and auxin induces root development in shoots to make it a complete plant. In the present study, in an attempt to induce multiple shoot regeneration of $D$. burmannii from shoot tips, these were cultured on different strengths of SM supplemented with different PGR concentrations (cytokinin and auxin). Shoot induction was observed on all the tested concentrations and combinations of PGR with varied response. The highest number of shoots $(28.8 \pm 1.5)$ was observed in one-fourth strength SM supplemented with $4 \mathrm{mg} / \mathrm{l}$ BAP and $4 \mathrm{mg} / \mathrm{l}$ NAA (Fig. 1; Table 1), which was followed by $26.8 \pm 1.4$ shoots in one-fourth strength SM fortified with $4 \mathrm{mg} / \mathrm{l} \mathrm{KN}$ and $4 \mathrm{mg} / \mathrm{l} \mathrm{NAA}$ (Table 2). The higher rate of multiple shoot induction in cultures supplemented with BAP and NAA may be largely ascribed to increased rates of cell division induced by cytokinin (BAP) in the terminal and axillary meristematic zone of explant tissues (Niranjan et al. 2010). The beneficiary effect of BAP and $\mathrm{KN}$ for the multiple shoot initiation has also been reported earlier (Jayaram and Prasad 2007). Considerably high number of shoots $(25.3 \pm 1.2)$ was also recorded in one-fourth strength SM supplemented with $1 \mathrm{mg} / \mathrm{l} \mathrm{Z}$ and $8 \mathrm{mg} / \mathrm{l} \mathrm{NAA}$ (Table 3). Most of the shoot tip explants cultured on different concentrations of MS supplemented with growth regulators grew in size and new shoots began to proliferate within 10-15 days of incubation.

Root formation was also observed on different strengths of SM with a combination of cytokinins and auxin. Although root induction was not observed on all the used concentrations and combinations of PGR, the maximum number of roots $(9.7 \pm 0.6)$ was observed on one-fourth strength SM supplemented with $4 \mathrm{mg} / \mathrm{l}$ BAP and $4 \mathrm{mg} / \mathrm{l}$ NAA. One-fourth strength SM supplemented with $\mathrm{BAP}+\mathrm{NAA}$ was found to be superior to $\mathrm{KN}+\mathrm{NAA}$ and 
$\mathrm{Z}+\mathrm{NAA}$ with respect to the induction of roots. The superiority of BAP + NAA over other PGR in in vitro propagation has also been reported in other plant species (Seyyedyousefi et al. 2013). Our data revealed that there are differences in the effect of the different concentrations of $\mathrm{BAP}+\mathrm{NAA}, \mathrm{KN}+\mathrm{NAA}$ and $\mathrm{Z}+\mathrm{NAA}$ on root induction.

One-way ANOVA of the experiment showed that there was a significant difference between the various concentrations of cytokinins and auxins and SM strength used in the study. Of the various treatments, one-fourth strength SM with BAP and NAA combination produced a maximum number of shoots and maximum root induction.

On the basis of our results, the effect of different concentrations of BAP, KN and $\mathrm{Z}$ in combination with NAA was evaluated; it was found that combination of BAP with NAA was found more suitable for shoot multiplication as well as root induction than $\mathrm{KN}$ with NAA and $\mathrm{Z}$ with NAA. Variation in the activity of cytokinins and auxins can be explained by differences in the uptake rate, respectively (Nikam and Savant 2007). The micropropagation protocol reported here was characterized with a rapid proliferation of shoots and rooting of $D$. burmannii. This method will be highly advantageous for large-scale production, as a uniform source of plants for commercial purposes, for pharmaceutical uses, for its conservation and a range of further biotechnological applications.

Drosera burmannii propagate mostly through seeds; however, very limited reports are available on seed germination and micropropagation. The present work demonstrated that it was possible to successfully obtain multiple shoots of $D$. burmannii from shoot tip cultures with an efficient in vitro rooting protocol. The establishment of protocols for in vitro propagation of $D$. burmannii through shoot multiplication provides a potential system for improvement, conservation and mass propagation of this amazing and medicinally important carnivorous plant.

Acknowledgements Funding was provided by the University Grants Commission.

\section{Compliance with ethical standards}

Conflict of interest The authors declare no conflict of interest.

\section{References}

Didry N, Dubreuil L, Trotin F, Pinkas M (1998) Antimicrobial activity of aerial parts of Drosera peltata Smith on oral bacteria. J Ethnopharmacol 60(1):91-96

Ellison AM, Gotelli NJ (2009) Energetics and the evolution of carnivorous plant-Darwin's 'most wonderful plants in the world'. J Exp Bot 60:19-42

Hema B, Bhupendra S, Saleem TM et al (2009) Anticonvulsant effect of Drosera burmannii Vahl. Int J Appl Res Nat Prod 2(3):1-4

Jayaram K, Prasad MNV (2006) Drosera indica L. and D. burmannii Vahl., medicinally important insectivorous plants in Andhra Pradesh-regional threats and conservation. Curr Sci 91(7):943-947

Jayaram K, Prasad MNV (2007) Rapid in vitro multiplication of Drosera indica L.: a vulnerable, medicinally important insectivorous plant. Plant Biotechnol Rep 1(2):79-84

Jennings DE, Krupa JJ, Raffel TR et al (2010) Evidence for competition between carnivorous plants and spiders. Proc R B Biol Sci 277:3001-3008

Joseph J, Joseph KM (1986) Insectivorous plants of Khasi and Jaintia hills, Meghalaya, India: a preliminary survey. Botanical Survey of India, Calcutta

McPherson S (2010) Carnivorous plants and their habitats, vol 2. Redfern Natural History, Poole, p 718

Moon DC, Rossi AM, Depaz J et al (2010) Ants provide nutritional and defensive benefits to the carnivorous plant Sarracenia minor. Oecol 164(1):185-192

Nikam TD, Savant RS (2007) Callus culture and micropropagation of Ceropegia sahyadrica Ans. and Kulk.: an edible starchy tuberous rare asclepiad. Indian J Plant Physiol 12(2):108-114

Niranjan MH, Sudarshana MS, Girisha ST (2010) In vitro multiple shoot induction from excised shoot tips and nodal segment explants of Lagerstroemia indica (L) - a medicinal cum ornamental shrub. J Biomed Sci Res 2:212-217

Nordbakken JF, Rydgren K, Okland RH (2004) Demography and population dynamics of Drosera anglica and D. rotundifolia. J Ecol 92(1):110-121

Ravikumar K, Ved DK, Vijaya SR et al (2000) 100 Red listed medicinal plants of conservation concern in Southern India. FRLHT, Bangalore, pp 261-263

Reddy CHS, Reddy KN, Jadhav SN (2001) Threatened (Medicinal) plants of Andhra Pradesh. Medicinal Plants Conservation Center, Hyderabad, pp 1-39

Seyyedyousefi SR, Kaviani B, Dehkaei NP (2013) The effect of different concentrations of NAA and BAP on micropropagation of Alstroemeria. Eur J Exp Biol 3(5):133-136

Su YH, Liu YB, Zhang XS (2011) Auxin-cytokinin interaction regulates meristem development. Mol Plant 4:616-625

Wagner H, Baldt S, Zgainski EM (1984) Plant drug analysis. Springer, Berlin, Heidelberg, New York

Wang Q, Su J, Zeng L (1998) The isolation and identification of flavonoids from Drosera burmannii. J Chin Med Mater 21(8):401-403 